\title{
PLANEJAMENTO DE REDES GEODÉSICAS RESISTENTES A MÚLTIPLOS OUTLIERS
}

\author{
Design of geodetic networks reliable against multiple outliers \\ IVANDRO KLEIN ${ }^{1,2}$ \\ MARCELO TOMIO MATSUOKA ${ }^{1,2}$ \\ SERGIO FLORENCIO DE SOUZA ${ }^{1,2}$ \\ CAROLINA COLLISCHONN ${ }^{1}$ \\ ${ }^{1}$ Laboratório de Pesquisas em Geodésia (LAGEO)/Departamento de \\ Geodésia/Instituto de Geociências - UFRGS \\ ${ }^{2}$ Programa de Pós-Graduação em Sensoriamento Remoto - UFRGS \\ Av. Bento Gonçalves, 9500, CEP 91501-970, \\ Cx. Postal 15001, Porto Alegre, RS, Brasil \\ ivandroklein@gmail.com; tomiomatsuoka@gmail.com; \\ sergioflorenciodesouza@gmail.com; carol.collischonn@gmail.com
}

\begin{abstract}
RESUMO
Ao se planejar o levantamento de uma rede geodésica, deseja-se que as observações a serem realizadas e as coordenadas dos pontos a serem estimadas atendam critérios de precisão e confiabilidade pré-estabelecidos de acordo com os objetivos do projeto. $\mathrm{Na}$ etapa de pré-análise, antes mesmo da coleta das observações, é possível estimar a precisão e confiabilidade da rede, estipulando uma geometria/configuração para a mesma e a precisão esperada para as observações. O objetivo deste artigo é apresentar o planejamento de uma rede geodésica que atenda critérios de precisão e confiabilidade, considerando a possível existência de dois ou mais erros não detectados nas observações, bem como a influência (simultânea) destes erros sobre os parâmetros (coordenadas ajustadas dos vértices). Além da revisão teórica, experimentos foram realizados em uma rede GNSS, onde foram estipulados critérios de precisão e confiabilidade considerando a existência de até duas observações contaminadas por erros (outliers), de maneira simultânea. O planejamento da rede foi feito por meio do método da tentativa e erro. Depois do processamento dos dados e do ajustamento da rede, se verificou que os critérios de precisão e confiabilidade que foram estipulados na etapa de pré-análise foram devidamente obtidos.
\end{abstract}

Palavras-chave: Redes Geodésicas; Pré-análise; Confiabilidade; Múltiplos Outliers. 
When planning a survey of a geodetic network, it is desirable that the observations to be measured and the points coordinates to be estimated follow criteria of precision and reliability preestablished according to the project objectives. In the pre-analysis step, even before the measurement of observations, it is possible to estimate the precision and reliability of the network, by setting a geometry / configuration for it and the expected precision of the observations. The goal of this paper is to present the design of a geodetic network following criteria of precision and reliability, taking into account the possible existence of two or more undetected errors in the observations, as well as their influence (simultaneous) on the parameters (adjusted coordinates of the points). Besides the theoretical review, experiments were performed in a GNSS network, that were established criteria of precision and reliability considering the existence of up to two observations contaminated by errors (outliers), simultaneously. The network design was done by the method of trial and error. After the data processing and the adjustment of the network, it was found that the criteria of accuracy and reliability that have been stipulated in the pre-analysis step were properly obtained.

Keywords: Geodetic Networks; Design; Reliability; Multiple Outliers.

\section{INTRODUÇÃO}

Uma rede geodésica é constituída por um conjunto de pontos materializados no terreno, com suas posições referenciadas a um sistema de coordenadas (TEIXEIRA; FERREIRA, 2003). Redes geodésicas são utilizadas nos mais diversos ramos da Ciência e da Engenharia, como por exemplo, na materialização de sistemas geodésicos de referência (ALTAMIMI et al., 2011), no monitoramento de deformações de estruturas (CHAVES, 2001), no cadastro técnico rural e urbano (AMORIM, 2004), em locação de obras de engenharia (PINTO, 2000), no monitoramento de fenômenos de natureza dinâmica sobre a superfície terrestre (PEREZ et al., 2003), dentre outros. Ao se projetar e levantar uma rede geodésica, deseja-se que as coordenadas estimadas para os pontos atinjam um critério de precisão pré-estabelecido de acordo com os objetivos do projeto, e, além disso, que se possa ter uma medida da dimensão da influência de possíveis erros não detectados nas observações sobre os valores estimados para as coordenadas dos pontos.

Desta forma, o planejamento ótimo de uma rede geodésica envolve uma série de fatores, como precisão, confiabilidade e custos (AMIRI-SIMKOOEI et al., 2012). $\mathrm{Na}$ etapa de pré-análise da rede, busca-se, antes da coleta dos dados, a otimização do levantamento, ou seja, projetar o levantamento geodésico de modo a obter resultados com a qualidade desejada, com as observações mais viáveis prática e economicamente (DALMOLIN, 2002). Sobre a qualidade da rede, Baarda (1977) propõe o termo "acurácia da rede geodésica", que consiste da "precisão da rede geodésica" (precisão das coordenadas dos vértices) e da "confiabilidade da rede geodésica" (relacionada aos testes estatísticos utilizados para localização de erros não aleatórios nas observações realizadas). 
Dentro deste contexto, uma série de trabalhos foram publicados sobre o planejamento (pré-análise) da precisão e da confiabilidade de redes geodésicas, como por exemplo, Baarda (1968, 1977), Grafarend (1974), Grafarend e Sanso (1985), Kuang (1996), Oliveira e Dalmolin (2003), Teixeira e Ferreira (2003), Monico et al. (2006), Oliveira (2007), Oliveira e Dalmolin (2008), Amiri-Simkooei et al. (2012).

Entretanto, as medidas de confiabilidade consideradas, inicialmente propostas por Baarda (1968), são relativas a testes estatísticos que consideram a existência de somente uma observação contaminada por erro por vez, como por exemplo, no caso do conhecido procedimento data snooping. Na prática, a estratégia adotada é repetir os testes estatísticos cada vez que uma nova observação suspeita é identificada.

Porém, segundo Gui et al. (2010), esta metodologia pode estar sujeita a algumas armadilhas, como o fenômeno masking (quando uma observação contaminada por erros é não detectada em virtude de outras observações também estarem contaminadas por erros), e também o fenômeno swamping (quando uma observação não contaminada por erros é identificada erroneamente devido a influência de observações contaminadas por erros).

Recentemente, medidas de confiabilidade para testes estatísticos que consideram a existência, simultânea, de duas ou mais observações contaminadas por erros (outliers) também foram propostas e investigadas (OBER, 1996; ANGUS, 2006; KNGHIT et al., 2010; ALMAGBILE et al., 2011; KLEIN et al., 2011).

Como na prática, duas ou mais observações podem estar contaminadas por erros, principalmente quando o número de observações é relativamente grande, o objetivo deste trabalho é apresentar o planejamento da precisão e confiabilidade de uma rede geodésica, considerando a possível existência de múltiplas observações contaminadas por erros. Além da revisão teórica, este artigo também apresenta experimentos realizados em uma rede GNSS (Global Navigation Satellite System), onde foram estipulados critérios de precisão e confiabilidade considerando a existência de até duas observações contaminadas por erros, de maneira simultânea. Por fim, os resultados obtidos são apresentados e discutidos.

\section{ACURÁCIA DE REDES GEODÉSICAS}

Para a estimação dos parâmetros incógnitos de uma rede geodésica (ou seja, as coordenadas dos pontos que constituem a rede), são realizadas observações geodésicas (como por exemplo, medidas de ângulos e distâncias entre pontos), e a estimação dos parâmetros incógnitos usualmente é realizada segundo o ajustamento destas observações pelo Método dos Mínimos Quadrados - MMQ (GHILANI; WOLF, 2006).

Quando apenas erros de natureza aleatória contaminam as observações, o ajustamento pelo MMQ é dito ser o melhor estimador linear imparcial para os parâmetros (TEUNISSEN, 2003). Entretanto, podem existir erros sistemáticos e/ou grosseiros nas observações, e desta forma, a influência destes erros pode contaminar os resultados do ajustamento (no caso, os valores estimados para as coordenadas dos pontos da rede). Portanto, é importante adotar uma estratégia de controle de 
qualidade dos resultados de um ajustamento. Seguindo esta linha de pensamento, de acordo com Baarda (1977), o conceito "acurácia de uma rede geodésica" consiste de duas partes: "precisão da rede geodésica" e "confiabilidade da rede geodésica".

A precisão da rede geodésica é dada pela matriz de covariância dos parâmetros estimados, obtida por meio da lei de propagação de variâncias e covariâncias das observações, enquanto a confiabilidade da rede geodésica diz respeito à detecção de erros (não aleatórios) nas observações, por meio de testes estatísticos, como por exemplo, a aplicação do método $\beta$, proposto por Baarda (1968). Neste método, o referido autor propõe o uso do Teste global do ajustamento para a detecção de erros nas observações e/ou no modelo, e o procedimento data snooping para a identificação (localização) das observações suspeitas de estarem contaminadas por erros, presumindo que somente uma observação está contaminada por vez, o que resulta em um processo iterativo de ajustamento e testes cada vez que uma nova observação suspeita é identificada e removida do modelo (KAVOURAS, 1982).

Desta forma, pode-se afirmar que, ao realizar o ajustamento pelo MMQ de uma rede geodésica, deseja-se que a precisão das coordenadas dos pontos (expressa pela matriz de covariância dos parâmetros estimados) atenda as necessidades do projeto para o qual esta rede foi designada, e ao mesmo tempo, que seja possível se ter uma estimativa da influência de possíveis erros remanescentes nas observações, sobre estas coordenadas, caso tais erros não sejam detectados pelo procedimento de teste utilizado.

Segundo Dalmolin e Oliveira (2010), conceitualmente, o trabalho do planejamento ótimo de uma rede geodésica é em geral, compreendido como a construção de uma configuração geométrica ótima e/ou de um plano de observações cujas realizações satisfaçam critérios de qualidade pré-definidos. Ainda segundo os mesmos autores, antes mesmo da etapa de coleta de observações em campo ser realizada, é possível o planejamento da rede geodésica de forma que estes critérios de qualidade pré-definidos sejam obedecidos, em uma etapa também conhecida como "pré-análise".

\section{PLANEJAMENTO (OU PRÉ-ANÁLISE) DE REDES GEODÉSICAS}

Quanto à precisão desejada para as coordenadas dos pontos de uma rede geodésica, os problemas de pré-análise podem ser agrupados em três casos gerais (DALMOLIN, 2002):

- Problemas de primeira ordem; quando é definida a matriz de covariância desejada para os parâmetros, bem como a matriz de covariância das observações, e deseja-se determinar (no caso do ajustamento pelo método paramétrico) a matriz design A, relacionada com a geometria/configuração da rede geodésica;

- Problemas de segunda ordem; quando é definida a matriz de covariância desejada para os parâmetros e a matriz design A, e deseja-se determinar a matriz de covariância das observações; 
- Problemas de terceira ordem; quando é definida somente a matriz de covariância desejada para os parâmetros, e deseja-se determinar a matriz design A e a matriz de covariância das observações.

Desta forma, na etapa de pré-análise da precisão de uma rede geodésica, é definida a matriz de covariância desejada para os parâmetros (ou seja, para as coordenadas estimadas dos pontos), e busca-se determinar a geometria/configuração da rede geodésica (expressa pela matriz design A), e/ou a precisão das observações (expressa pela matriz de covariância das observações), que resulte nesta precisão desejada para os parâmetros. A matriz de covariância dos parâmetros estimados $\left(\Sigma_{X}\right)$ pode ser obtida em função da matriz design A e da matriz de covariância das observações $\left(\Sigma_{L}\right)$, por meio da lei de propagação de variâncias e covariâncias das observações sobre os parâmetros, resultando em (GHILANI; WOLF, 2006):

$$
\Sigma_{X}=\sigma_{0}^{2}\left(A^{T} P A\right)^{-1},
$$

onde na expressão (1), $\sigma_{0}^{2}$ é o fator de variância a priori (de caráter arbitrário), e $\mathrm{P}$ é a matriz peso das observações, usualmente tomada como sendo igual à $P=\sigma_{0}^{2} \sum_{L}^{-1}$ (GEMAEL, 1994).

Desta forma, os problemas de pré-análise podem ser resolvidos por meio de duas maneiras: pela solução analítica (ou ótima) da expressão (1), ou pelo método heurístico, também conhecido como tentativa e erro (AMIRI-SIMKOOEI et al., 2012). Segundo Dalmolin (2002), na prática, a solução analítica só se aplica aos problemas de segunda ordem e aos casos mais simples da pré-análise. Para os problemas mais complexos, a solução mais abrangente e viável é por meio do método da tentativa e erro, ou seja, estipular uma matriz design A e uma matriz de covariância para as observações $\left(\Sigma_{L}\right)$, e verificar se a matriz de covariância resultante para os parâmetros, obtida por meio da expressão (1), satisfaz os critérios de precisão que foram pré-estabelecidos.

Importante ressaltar que existem outras classificações para os problemas de otimização de redes geodésicas, como no clássico trabalho de Grafarend (1974), que agrupa o planejamento de redes geodésicas em quatro casos gerais: Problemas de ordem zero (definição ótima do datum), problemas de primeira ordem (design de uma geometria/configuração ótima para a rede), problemas de segunda ordem (otimização dos pesos das observações), e problemas de terceira ordem (densificação ótima de uma rede já existente, adicionando novas observações e/ou vértices).

Quanto à confiabilidade da rede geodésica, a mesma pode ser estimada por meio da confiabilidade interna e da confiabilidade externa. A confiabilidade interna quantifica o menor erro detectável em uma observação, segundo o procedimento de teste utilizado e os níveis de probabilidade assumidos, e a confiabilidade externa 
estima a influência deste erro nos resultados do ajustamento (ou seja, nos parâmetros estimados), quando não detectado (OLIVEIRA; DALMOLIN, 2008).

Como as medidas de confiabilidade interna e externa dependem, dentre outros fatores, da matriz de covariância das observações e da matriz design A, e, além disso, independem do valor numérico das observações, estas medidas também podem ser estimadas antes da coleta das observações, ou seja, na etapa de préanálise (para maiores detalhes, ver MONICO et al., 2006 e KLEIN, 2012).

Entretanto, salienta-se que as medidas de confiabilidade convencionais (BAARDA, 1968; BAARDA, 1977; KAVOURAS, 1982; FÖRSTNER, 1987) foram desenvolvidas para os procedimentos de teste convencionais, como por exemplo, o data snooping, que pressupõem que apenas uma observação está contaminada por erro por vez. Na prática, porém, mais de uma observação pode conter erros (não aleatórios), principalmente quando o número de observações é relativamente grande (ALMAGBILE et al., 2011).

Desta forma, se forem utilizadas as chamadas medidas de confiabilidade convencionais na etapa de planejamento de uma rede geodésica, pode-se estimar o menor erro detectável de cada observação, bem como as influências destes nos resultados do ajustamento (quando não detectados), mas, considerando que apenas uma única observação está contaminada por um erro (não aleatório) por vez.

Porém, se for desejado o planejamento de uma rede geodésica, de forma que se possa estimar a influência máxima sobre os parâmetros, de duas ou mais observações contaminadas por erros (não detectados), de maneira simultânea, devese utilizar as medidas de confiabilidade generalizadas para estes casos (ver, por exemplo, KNIGHT et al., 2010).

\subsection{Planejamento de uma Rede Geodésica Resistente a Múltiplos Outliers}

A confiabilidade interna de uma observação, na abordagem generalizada para múltiplos outliers, estima o menor erro detectável (mininal detectable bias - MDB) desta observação, considerando que, além dela, outras observações também estão contaminadas por erros (não aleatórios), de maneira simultânea. Entretanto, para estes casos, ressalva-se que cada uma das n observações terá $\left(\begin{array}{l}n-1 \\ q-1\end{array}\right)$ confiabilidades internas (MDB) possíveis, estipulado o número de outliers “q” considerado, ou seja, o número de observações consideradas, simultaneamente, suspeitas (KNIGHT et al., 2010).

Como na prática, geralmente se está mais interessado na dimensão da influência de possíveis erros não detectados nas observações sobre os resultados do ajustamento (ou seja, sobre os parâmetros que foram estimados, como as coordenadas dos pontos de uma rede geodésica), na etapa de pré-análise da confiabilidade da rede, considerando a abordagem para múltiplos outliers, pode ser utilizado como critério somente a confiabilidade externa para múltiplos outliers, 
tendo em vista o alto número de cálculos envolvidos na confiabilidade interna para estes casos (ver, por exemplo, ALMAGBILE et al., 2011).

A confiabilidade externa generalizada para múltiplos outliers estima a influência máxima de duas ou mais observações contaminadas por erros (não detectados), de maneira simultânea, sobre apenas um parâmetro específico do vetor dos parâmetros estimados (KLEIN et al., 2011).

A influência máxima ( $\nabla x_{k}^{q}-$ confiabilidade externa) de q observações contaminadas por erros (com q $>1$ ), sobre um parâmetro específico $\left(x_{k}\right)$ do vetor dos parâmetros estimados X, é dada por (OBER, 1996):

$$
\nabla x_{k}^{q}=\sqrt{\lambda_{\text {máx }}}
$$

sendo que $\lambda_{\text {máx }}$ corresponde ao autovalor máximo do problema generalizado de autovalores e autovetores (KNIGHT et al., 2010):

$$
\left(\lambda_{0}\left(C_{L}^{T} \sum_{L}^{-1} \sum_{V} \sum_{L}^{-1} C_{L}\right)^{-1} C_{L}^{T} \sum_{L}^{-1} A\left(A^{T} P A\right)^{-1} c_{x_{k}}^{T} C_{x_{k}}\left(A^{T} P A\right)^{-1} A^{T} P C_{L}\right) u=\lambda u
$$

Na expressão (3), $\lambda_{0}$ corresponde ao parâmetro de não centralidade do modelo (TEUNISSEN, 2006), obtido em função do número de outliers q considerado, e dos níveis de probabilidade assumidos (poder e nível de significância do teste), $C_{L}$ é uma matriz de dimensão n por q, referente ao modelo de erro adotado, ou seja, quais são as observações suspeitas consideradas (ver, por exemplo, KLEIN et al., 2011), $\Sigma_{V}$ é a matriz de covariância dos resíduos estimados (ou seja, do vetor V), obtida em função da lei de propagação de variâncias e covariâncias, e dependente, essencialmente, da matriz de covariância das observações e da matriz design A, e $c_{X_{k}}$ é um vetor unitário de dimensão u por 1 (sendo u igual ao número de parâmetros incógnitos do modelo), contendo a unidade na linha do k-ésimo parâmetro ao qual se busca obter a confiabilidade externa máxima das observações suspeitas consideradas, e zeros nas demais linhas (ver, por exemplo, KNIGHT et al., 2010; KLEIN, 2012).

Analisando a expressão (3), nota-se que a confiabilidade externa máxima de duas ou mais observações sobre um parâmetro qualquer depende, essencialmente, da geometria/configuração do problema (expressa pela matriz design A), da precisão e eventual correlação das observações (expressa pela matriz de covariância das observações, e, consequentemente, pela matriz peso), do número de outliers q considerado e dos níveis de probabilidade assumidos para o teste (dos quais se obtêm o valor do parâmetro de não centralidade do modelo). Ou seja, esta medida independe do valor numérico das observações, podendo também ser estimada antes da etapa de campo, tal como a matriz de covariância dos parâmetros ajustados 
(expressão 1), bastando para isso, estipular uma matriz design A (configuração para a rede), a precisão esperada para as observações, o número de outliers simultâneos considerados (q) e os níveis de probabilidade assumidos para o teste estatístico de identificação de erros em questão.

Portanto, fixando o número de outliers q considerado e os níveis de probabilidade assumidos para o teste de identificação de erros (nível de significância e poder do teste), por meio do método de tentativa e erro, pode-se determinar uma geometria para a rede (matriz design A) e/ou uma matriz de covariância adequada para as observações, que atenda aos critérios de confiabilidade pré-estabelecidos, no caso, a influência máxima de possíveis q observações contaminadas por erros (não detectados), sobre cada um dos parâmetros incógnitos do modelo a serem estimados.

Além disso, ressalva-se que para cada parâmetro $x_{k}$, tem-se $\left(\begin{array}{l}n \\ q\end{array}\right)$ confiabilidades externas associadas, uma para cada combinação possível da matriz $C_{L}$, estipulado o número de outliers q considerado. Uma discussão mais detalhada sobre o tema é obtida em Knight et al. (2010).

Finalmente, também se faz necessário apresentar o teste estatístico relativo a estas medidas de confiabilidade generalizadas, que consideram a existência, simultânea, de duas ou mais observações contaminadas por erros (não aleatórios), ao contrário do procedimento de teste convencional data snooping (onde apenas uma observação é considerada suspeita por vez).

O teste geral para a identificação de erros, assumindo que mais do que uma observação, simultaneamente, esteja contaminada por erros grosseiros (outliers), testa as seguintes hipóteses (TEUNISSEN, 2006):

$$
H_{0}: E\left\{L_{n x 1}\right\}=A_{n x u} X_{u x 1}
$$

contra

$$
H_{A}: E\left\{L_{n x 1}\right\}=A_{n x u} X_{u x 1}+C_{L n x q} \nabla_{q x 1}, \nabla_{q x 1} \neq 0_{q x 1}
$$

onde $L_{n x 1}$ é o vetor das n observações (medidas) realizadas, $X_{u x 1}$ é o vetor dos u parâmetros incógnitos a serem estimados, $C_{L_{n \times q}}$ é uma matriz de dimensão n por q, referente ao modelo de erro adotado, ou seja, quais são as observações suspeitas consideradas, e $\nabla_{q \times 1}$ é o vetor dos q erros grosseiros correspondentes (cada um relativo a uma das q observações suspeitas).

$\mathrm{Na}$ hipótese nula considerada na expressão (4), apenas erros aleatórios contaminam o vetor das observações, e na hipótese alternativa, as q observações suspeitas estão contaminadas por q erros (não aleatórios), de maneira simultânea. $\mathrm{O}$ critério de teste é dado por (TEUNISSEN, 2006): 
Rejeitar $\mathrm{H}_{0}$ se:

$$
T_{q}>\chi_{\alpha}^{2}
$$

onde a estatística de teste $T_{q}$ em (5) é obtida por meio da seguinte expressão (TEUNISSEN, 2006):

$$
T_{q}=V^{T} \sum_{L}^{-1} C_{L}\left(C_{L}^{T} \sum_{L}^{-1} \sum_{V} \Sigma_{L}^{-1} C_{L}\right)^{-1} C_{L}^{T} \sum_{L}^{-1} V
$$

e $\chi_{\alpha}^{2}$ na expressão (5) corresponde ao valor tabelado para a distribuição qui quadrado, com q graus de liberdade e o nível de significância $\alpha$ que foi assumido para o teste definido em (4).

Como na prática, o verdadeiro número de outliers que contaminam o vetor das observações é sempre desconhecido, segundo Knight et al. (2010), o procedimento é aplicar a estatística de teste em (6) para $q=1$ e determinar a observação suspeita mais provável, com base no pressuposto de apenas um outlier. Em seguida, a estatística de teste em (6) é aplicada para q=2, e o par de observações suspeitas que apresentar o valor máximo para esta será o mais provável, com base no pressuposto de dois outliers simultâneos. Este processo é, então, realizado até q ser igual ao número máximo de outliers razoavelmente considerados (tendo em vista que $1 \leq q \leq n-u)$. Assim, as observações suspeitas identificadas, com base na variação do número de outliers q considerado, são então usadas como ponto de partida para novas investigações.

Ressalva-se que o teste generalizado para múltiplos outliers simultâneos, assim como o procedimento data snooping, também é sujeito a algumas limitações (como os já mencionados fenômenos swamping e masking, por exemplo), além de ter um alto custo computacional envolvido. Uma discussão mais detalhada sobre o tema pode ser obtida em Teunissen (2006), Knight et al. (2010) e Klein (2012).

\section{EXPERIMENTOS REALIZADOS}

Visando apresentar na prática o planejamento de uma rede geodésica resistente a múltiplos outliers (simultâneos), um estudo foi realizado utilizando dados de estações da Rede Brasileira de Monitoramento Contínuo (RBMC). No caso deste estudo, foi assumido que as estações PPTE (Presidente Prudente - SP), SPAR (Araçatuba - SP), ILHA (Ilha Solteira - SP) e OURI (Ourinhos - SP) seriam os vértices desconhecidos da rede geodésica a ser levantada, cujas coordenadas cartesianas geocêntricas (X, Y, Z) devem ser determinadas. As estações SJRP (São José do Rio Preto - SP) e ROSA (Rosana - SP) seriam os pontos de controle da rede, considerando as coordenadas oficiais destas estações (no referencial 
SIRGAS2000, homologadas pelo IBGE) como constantes (injunções absolutas) no processo de ajustamento.

Desta forma, o seguinte cenário foi simulado: Sobre a precisão da rede, desejase que as coordenadas estimadas para os quatro vértices desconhecidos apresentem um desvio-padrão de no máximo $5 \mathrm{~cm}$, e sobre a confiabilidade da rede, deseja-se que possíveis erros remanescentes (não detectados), em até duas observações, exerçam, simultaneamente, uma influência de no máximo $15 \mathrm{~cm}$ em cada uma das coordenadas ajustadas de cada um dos quatro vértices desconhecidos. Ou seja, tratase de um problema de planejamento (pré-análise) da acurácia (precisão e confiabilidade) de uma rede geodésica, que será resolvido por meio do método da tentativa e erro.

O valor para a confiabilidade externa máxima de duas observações sobre cada parâmetro $(15 \mathrm{~cm})$ foi arbitrado como sendo três vezes maior que o desvio-padrão máximo desejado para estes parâmetros $(5 \mathrm{~cm})$, pois o menor erro detectável em cada observação, em geral, é relativamente maior do que o desvio-padrão desta observação. Por exemplo, para redes geodésicas que possuem um número de observações (n) igual ao dobro do número de parâmetros (u), o menor erro detectável de uma observação, será, em média, igual a 5,8 vezes o desvio padrão desta observação, no caso do procedimento data snooping, ao nível de significância $\alpha_{0}=0,001$ e um poder do teste de $\gamma=0,80$ (KAVOURAS, 1982). Além disso, o menor erro detectável em uma observação, e consequentemente a sua influência sobre os parâmetros, em geral, aumenta (ou no melhor caso permanece o mesmo), conforme se aumenta o número de observações suspeitas (simultaneamente) consideradas (KNIGHT et al., 2010; KLEIN, 2012). Portanto, a tolerância assumida para a confiabilidade das observações realizadas, especialmente na abordagem para múltiplos outliers, deve ser maior do que a tolerância assumida para a precisão dos parâmetros a serem estimados.

Para o caso deste problema (ajustamento de uma rede GNSS), as coordenadas cartesianas geocêntricas $(X, Y, Z)$ dos vértices desconhecidos PPTE, SPAR, ILHA e OURI são os $(\mathrm{u}=4 \times 3=12)$ parâmetros da rede a serem estimados, enquanto as componentes das linhas-base independentes (distâncias relativas $\Delta \mathrm{X}, \Delta \mathrm{Y}$ e $\Delta \mathrm{Z}$ entre pares de vértices da rede), serão as n observações a serem levantadas por meio da técnica de posicionamento relativo, e, posteriormente, ajustadas (para maiores detalhes, ver MONICO, 2008).

Portanto, este problema de pré-análise consiste em: Quantas (e quais) linhasbase devem ser levantadas (configuração da rede, expressa pela matriz design A), e qual a precisão necessária para as observações de cada linha-base (expressa pela matriz de covariância das observações), de forma que o desvio-padrão das coordenadas dos quatro vértices desconhecidos (estimados) seja de no máximo 5 $\mathrm{cm}$, e a possível existência de erros remanescentes (não detectados), em até duas observações, exerça uma influência (simultânea/conjunta) de no máximo $15 \mathrm{~cm}$ sobre cada coordenada de cada vértice desconhecido da rede. Desta forma, sobre a 
precisão da rede, segundo a classificação de Dalmolin (2002), este seria um problema de terceira ordem.

Adotando o método da tentativa e erro, e considerando os custos envolvidos no projeto, em um primeiro cenário, foi simulado o levantamento de nove linhas-base independentes entre os vértices da rede, conforme ilustra a Figura 1.

Figura 1 - Configuração da geometria da rede, com nove linhas-base independentes.

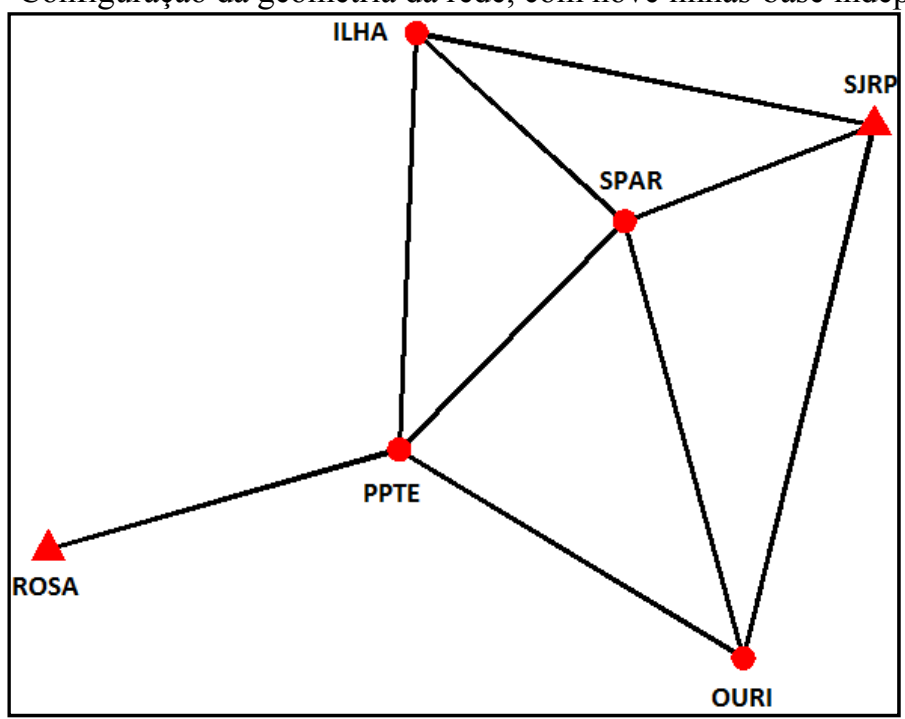

Para estas nove linhas-base (PPTE - SPAR, PPTE - ILHA, PPTE - OURI, SJRP - SPAR, SJRP - ILHA, SJRP - OURI, SPAR - ILHA, SPAR - OURI, ROSA - PPTE), a estratégia de ponderação para a precisão esperada das observações foi baseada nas seguintes considerações: De acordo com Monico (2008), o posicionamento relativo pode fornecer uma precisão entre 0,1 e 1 ppm (partes por milhão), ou até melhor do que isso, devendo, para o caso de linhas-base longas (maiores do que $20 \mathrm{~km}$ ), ser imprescindível o uso de receptores GNSS de dupla frequência. Neste caso, todas as linhas-base são longas (entre 120 e $250 \mathrm{~km}$ ), e como as estações são pertencentes à RBMC, todas elas possuem receptores de dupla frequência rastreando dados GNSS de maneira contínua. Além disso, de acordo com IBGE (2008), para linhas-base maiores do que $100 \mathrm{Km}$, o tempo de rastreio mínimo em cada linha-base (par de vértices ocupado simultaneamente) deve ser de $4 \mathrm{~h}$. Assim sendo, considerou-se uma precisão esperada de: $\sigma_{\text {linha-base }}=0,5 \mathrm{ppm}$ (valor intermediário entre 0,1 e 1 ppm), planejando tempos de rastreio de $6 \mathrm{~h}$ (duas horas a mais do que o mínimo recomendado pelo referido manual do IBGE).

Salienta-se que poderia ser utilizada outra estratégia de ponderação inicial, como por exemplo, com base no manual do fabricante do equipamento. Das seis 
estações, cinco são do fabricante Trimble (modelos NetRS, NetR8 e NetR5) e um do fabricante Leica (modelo GRX1200GGPRO). Consultando os manuais dos modelos, encontrou-se valores de precisão da ordem de $1 \mathrm{ppm}$ para os receptores Trimble. Entretanto, estes valores são relativos ao posicionamento relativo cinemático em tempo real (RTK - Real Time Kinematic), não sendo informados os valores para o posicionamento estático pós-processado. Para o receptor Leica, encontrou-se um valor da ordem de 0,5 ppm para o posicionamento estático pósprocessado. Como neste estudo, trata-se de posicionamentos estáticos, pósprocessados e com $6 \mathrm{~h}$ de ocupação, preferiu-se adotar a referida estratégia de ponderação (0,5 ppm x comprimento da linha-base).

Desta forma, a Tabela 1 apresenta o comprimento das nove linhas-base, bem como a precisão esperada para cada componente $(\Delta \mathrm{X}, \Delta \mathrm{Y}$ e $\Delta \mathrm{Z})$ destas, seguindo esta estratégia de ponderação.

Tabela 1 - Comprimento de cada linha-base e a precisão esperada em suas componentes (observações).

\begin{tabular}{c|c|c}
\hline Linha-Base & Comprimento & Precisão esperada em $\Delta \mathbf{X}, \Delta \mathbf{Y} \mathbf{e} \Delta \mathbf{Z}$ \\
\hline PPTE - SPAR & $144,155 \mathrm{Km}$ & $4,2 \mathrm{~cm}$ \\
\hline PPTE - ILHA & $187,480 \mathrm{Km}$ & $5,4 \mathrm{~cm}$ \\
\hline PPTE - OURI & $180,762 \mathrm{Km}$ & $5,2 \mathrm{~cm}$ \\
\hline SJRP - SPAR & $120,673 \mathrm{Km}$ & $3,5 \mathrm{~cm}$ \\
\hline SJRP - ILHA & $210,517 \mathrm{Km}$ & $6,1 \mathrm{~cm}$ \\
\hline SJRP - OURI & $245,882 \mathrm{Km}$ & $7,1 \mathrm{~cm}$ \\
\hline SPAR - ILHA & $125,984 \mathrm{Km}$ & $3,6 \mathrm{~cm}$ \\
\hline SPAR - OURI & $203,319 \mathrm{Km}$ & $5,9 \mathrm{~cm}$ \\
\hline ROSA - PPTE & $165,187 \mathrm{Km}$ & $4,8 \mathrm{~cm}$ \\
\hline
\end{tabular}

Nesta estratégia de ponderação, foi considerada uma precisão (desvio-padrão $\sigma)$ igual para cada componente: $\sigma=\sigma_{\text {linha-base }} / \sqrt{3}$, embora em um planejamento mais rigoroso, se pudesse ponderar cada observação $(\Delta \mathrm{X}, \Delta \mathrm{Y}$ e $\Delta \mathrm{Z})$ em função de seu próprio comprimento, ao invés de utilizar o comprimento resultante da linha-base, como foi realizado. Salienta-se que no método da tentativa e erro, conforme o próprio termo já diz, o geodesista está livre para definir os seus critérios de planejamento da forma que julgar adequado (correndo, evidentemente, o risco de não atingir os objetivos que foram pré-estabelecidos se os seus critérios forem significativamente discrepantes da realidade). Importante ressaltar também que, em redes GNSS, em função dos diversos tipos de erros envolvidos nas observáveis GNSS (ver, por exemplo, MONICO, 2008), é mais difícil estimar uma precisão $a$ priori para as observações do que em outros tipos de redes geodésicas (poligonais topográficas, redes de nivelamento geométrico, circuitos gravimétricos, 
fototriangulações, redes de triangulação ou trilateração geodésicas e etc.), onde geralmente, é possível obter uma boa estimativa para a precisão das observações antes da etapa de campo, em virtude somente do equipamento e da técnica de observação utilizada.

Definidas as nove linhas-base (considerando os vértices SJRP e ROSA como pontos de controle), e a precisão esperada para cada observação de cada linha-base (desconsiderando a eventual correlação entre estas), se têm uma matriz design A e uma matriz de covariância a priori das observações. Considerando a matriz peso como $P=\sum_{L}^{-1}$ (ou seja, arbitrando $\sigma_{0}^{2}=1$ ), por meio da expressão (1), pode-se estimar uma matriz de covariância a priori para os parâmetros. Com as variâncias e covariâncias estimadas para os parâmetros desta maneira, pode-se então obter os desvios-padrão esperados para os parâmetros, conforme apresenta a Tabela 2:

Tabela 2 - Precisão esperada para as coordenadas $(\mathrm{X}, \mathrm{Y}, \mathrm{Z})$ dos quatro vértices desconhecidos da rede GNSS.

\begin{tabular}{c|c|c}
\hline Vértice & Parâmetro (coordenada) & Precisão esperada (em cm) \\
\hline \multirow{4}{*}{ PPTE } & $\mathbf{X}_{\text {PPTE }}$ & 3,1 \\
\cline { 2 - 3 } & $\mathbf{Y}_{\text {PPTE }}$ & 3,1 \\
\cline { 2 - 3 } & $\mathbf{Z}_{\text {PPTE }}$ & 3,1 \\
\hline \multirow{4}{*}{ SPAR } & $\mathbf{X}_{\text {SPAR }}$ & 2,7 \\
\cline { 2 - 3 } & $\mathbf{Y}_{\text {SPAR }}$ & 2,7 \\
\cline { 2 - 3 } & $\mathbf{Z}_{\text {SPAR }}$ & 2,7 \\
\hline \multirow{4}{*}{ ILHA } & $\mathbf{X}_{\text {ILHA }}$ & 3,4 \\
\cline { 2 - 3 } & $\mathbf{Y}_{\text {ILHA }}$ & 3,4 \\
\cline { 2 - 3 } & $\mathbf{Z}_{\text {ILHA }}$ & 3,4 \\
\hline \multirow{4}{*}{ OURI } & $\mathbf{X}_{\text {OURI }}$ & 3,9 \\
\cline { 2 - 3 } & $\mathbf{Y}_{\text {OURI }}$ & 3,9 \\
\cline { 2 - 3 } & $\mathbf{Z}_{\text {OURI }}$ & 3,9 \\
\hline
\end{tabular}

Analisando os resultados apresentados na Tabela 2, nota-se que com estas nove linhas-base (Figura 1) e com esta precisão esperada para as observações $(\sigma=$ $\sigma_{\text {linha-base }} / \sqrt{3}$ ), o critério de planejamento da precisão da rede geodésica (coordenadas dos pontos com um desvio padrão de no máximo $5 \mathrm{~cm}$ ) é satisfeito. Porém, resta ainda verificar a confiabilidade da rede geodésica neste primeiro cenário de planejamento, com nove linhas-base independentes.

Considerando o valor para o parâmetro de não centralidade do modelo na expressão (3) como sendo: $\lambda_{0}=17,075$, valor este obtido em função dos níveis de probabilidade (nível de significância e poder do teste) e do número de graus de 
liberdade do teste utilizado para identificação de erros (para maiores detalhes, ver BAARDA, 1968; KAVOURAS, 1982; TEUNISSEN, 2006; KNIGHT et al., 2010; KLEIN et al., 2011; KLEIN, 2012), pode-se resolver o problema de autovalores e autovetores da expressão (3) para estimar a influência máxima de possíveis erros não detectados em cada par de observações sobre cada parâmetro. Para este cenário, onde o número de observações é $\mathrm{n}=3 \times 9=27$ (componentes $\Delta \mathrm{X}, \Delta \mathrm{Y}$ e $\Delta \mathrm{Z}$ das nove linhas-base independentes), e o número de observações suspeitas (simultaneamente) consideradas é $\mathrm{q}=2$, tem-se $\left(\begin{array}{l}n \\ q\end{array}\right)=\left(\begin{array}{c}27 \\ 2\end{array}\right)=351$ confiabilidades externas possíveis para cada um dos $(u=12)$ parâmetros incógnitos da rede. Desta forma, a Tabela 3 apresenta o par de observações que resulta na confiabilidade externa máxima sobre cada parâmetro (das 351 combinações possíveis para cada um destes, com $q=2$ ), bem como os valores destas influências máximas sobre cada parâmetro.

Tabela 3 - Pares de observações com confiabilidade externa máxima sobre cada parâmetro da rede.

\begin{tabular}{|c|c|c|c|}
\hline Ponto & Coordenada & $\begin{array}{c}\text { Par de observações com } \\
\text { confiabilidade externa máxima }\end{array}$ & $\begin{array}{c}\text { Influência } \\
\text { máxima }\end{array}$ \\
\hline \multirow{3}{*}{ PPTE } & $\mathbf{X}_{\mathrm{PPTE}}$ & $\Delta \mathrm{X}$ (SJRP - SPAR) e $\Delta \mathrm{X}$ (ROSA - PPTE) & $19 \mathrm{~cm}$ \\
\hline & $\mathbf{Y}_{\text {PPTE }}$ & $\Delta \mathrm{Y}(\mathrm{SJRP}$ - SPAR) e $\Delta \mathrm{Y}$ (ROSA - PPTE) & $19 \mathrm{~cm}$ \\
\hline & $\mathbf{Z}_{\mathrm{PPTE}}$ & $\Delta \mathrm{Z}$ (SJRP - SPAR) e $\Delta \mathrm{Z}$ (ROSA - PPTE) & $19 \mathrm{~cm}$ \\
\hline \multirow{3}{*}{ SPAR } & $\mathbf{X}_{\text {SPAR }}$ & $\triangle \mathrm{X}(\mathrm{SJRP}$ - SPAR) e $\Delta \mathrm{X}$ (ROSA - PPTE) & $19,2 \mathrm{~cm}$ \\
\hline & $\mathbf{Y}_{\text {SPAR }}$ & $\Delta \mathrm{Y}(\mathrm{SJRP}$ - SPAR) e $\Delta \mathrm{Y}$ (ROSA - PPTE) & $19,2 \mathrm{~cm}$ \\
\hline & $\mathbf{Z}_{\mathrm{SPAR}}$ & $\Delta \mathrm{Z}$ (SJRP - SPAR) e $\Delta \mathrm{Z}$ (ROSA - PPTE) & $19,2 \mathrm{~cm}$ \\
\hline \multirow{3}{*}{ ILHA } & $\mathbf{X}_{\text {ILHA }}$ & $\Delta \mathrm{X}(\mathrm{SJRP}$ - ILHA) e $\Delta \mathrm{X}$ (SPAR - ILHA) & $22,2 \mathrm{~cm}$ \\
\hline & $\mathbf{Y}_{\text {ILHA }}$ & $\Delta \mathrm{Y}(\mathrm{SJRP}$ - ILHA) e $\Delta \mathrm{Y}$ (SPAR - ILHA) & $22,2 \mathrm{~cm}$ \\
\hline & $\mathbf{Z}_{\text {ILHA }}$ & $\Delta \mathrm{Z}$ (SJRP - ILHA) e $\Delta \mathrm{Z}$ (SPAR - ILHA) & $22,2 \mathrm{~cm}$ \\
\hline \multirow{3}{*}{ OURI } & $\mathbf{X}_{\text {OURI }}$ & $\Delta \mathrm{X}$ (PPTE - OURI) e $\Delta \mathrm{X}(\mathrm{SPAR}$ - OURI) & $24,4 \mathrm{~cm}$ \\
\hline & $\mathbf{Y}_{\text {OURI }}$ & $\Delta \mathrm{Y}(\mathrm{PPTE}-\mathrm{OURI})$ e $\Delta \mathrm{Y}$ (SPAR - OURI) & $24,4 \mathrm{~cm}$ \\
\hline & $\mathbf{Z}_{\text {OURI }}$ & $\Delta \mathrm{Z}$ (PPTE - OURI) e $\Delta \mathrm{Z}$ (SPAR - OURI) & $24,4 \mathrm{~cm}$ \\
\hline
\end{tabular}

Analisando a Tabela 3, nota-se que nenhuma coordenada de nenhum dos quatro vértices incógnitos apresenta uma confiabilidade externa (máxima) inferior a $15 \mathrm{~cm}$, ou seja, o critério de planejamento da confiabilidade da rede geodésica (influência máxima de possíveis erros não detectados em até duas observações, sobre cada parâmetro, inferior a $15 \mathrm{~cm}$ ) não é satisfeito. Desta forma, pelo método da tentativa e erro, deve-se melhorar a precisão das observações e/ou a 
geometria/configuração da rede, para que este critério de confiabilidade préestabelecido seja satisfeito.

Neste caso, devido a impossibilidade de estimar (com alta confiança) uma precisão a priori para as observações da rede GNSS, em virtude dos diversos tipos de erros envolvidos (além da correlação entre as componentes de uma mesma linhabase), optou-se pela segunda opção, ou seja, aumentar o número de linhas-base (observações) da rede, aumentando assim a redundância (graus de liberdade) do ajustamento, bem como alterando a geometria/configuração da mesma.

Para aumentar o número de linhas-base, duas opções podem ser consideradas: Ocupar novas linhas-base, ou, "repetir" linhas-base que já foram consideradas. Como as distâncias entre os vértices desta rede são relativamente longas (maiores que $120 \mathrm{Km}$ ), considerando também os custos e o tempo de execução do projeto, optou-se por considerar duas ocupações independentes em algumas das linhas-base da rede, considerando que a confiabilidade das observações de linhas-base que são ocupadas duas ou mais vezes melhora consideravelmente (KLEIN, 2012). Seguindo a metodologia da tentativa e erro, três linhas-base foram escolhidas para serem ocupadas duas vezes: SPAR - ILHA, SPAR - OURI e SJRP - SPAR. O motivo destas escolhas é porque estas são algumas das linhas-base que constam nos pares de observações que apresentam confiabilidade externa máxima na Tabela 3, além de conter o vértice central da rede (SPAR), sendo que a localização dos vértices está diretamente relacionada com a propagação de erros (ver, por exemplo, KLEIN, 2012). Além disso, os vértices ILHA e OURI apresentaram uma influência maior em suas coordenadas do que o vértice PPTE (ver a Tabela 3).

Para as três linhas-base "repetidas" que foram incluídas na rede (ILHA SPAR, OURI - SPAR, SPAR - SJRP), a estratégia de ponderação da precisão das observações foi a mesma adotada para as demais (ou seja, de $0,5 \mathrm{ppm}$, considerando um tempo de rastreio de $6 \mathrm{~h}$ ). Portanto, a precisão esperada para as componentes $\Delta \mathrm{X}$, $\Delta \mathrm{Y}$ e $\Delta \mathrm{Z}$ destas linhas-base é igual à precisão esperada para as componentes $\Delta \mathrm{X}$, $\Delta \mathrm{Y}$ e $\Delta \mathrm{Z}$ de SPAR - ILHA, SPAR - OURI e SJRP - OURI, respectivamente (ver a Tabela 1).

Definidas as doze linhas-base (novamente considerando os vértices SJRP e ROSA como pontos de controle), e a precisão esperada para cada observação de cada linha-base, se têm uma nova matriz design A e uma nova matriz de covariância a priori das observações (novamente, desconsiderando as eventuais correlações entre estas). Mantendo a definição da matriz peso como $P=\sum_{L}{ }^{-1}$ (ou seja, arbitrando $\sigma_{0}^{2}=1$ ), por meio da expressão (1), pode-se estimar uma nova matriz de covariância a priori para os parâmetros. Com as novas variâncias e covariâncias estimadas para os parâmetros desta maneira, pode-se então obter os desvios-padrão esperados para os parâmetros neste segundo cenário, conforme apresenta a Tabela 4: 
Tabela 4 - Precisão esperada para as coordenadas dos quatro vértices desconhecidos da rede GNSS (com as três linhas-base adicionais).

\begin{tabular}{c|c|c}
\hline Vértice & Parâmetro (coordenada) & Precisão esperada (em cm) \\
\hline \multirow{4}{*}{ PPTE } & $\mathbf{X}_{\text {PPTE }}$ & 2,9 \\
\cline { 2 - 3 } & $\mathbf{Y}_{\text {PPTE }}$ & 2,9 \\
\cline { 2 - 3 } & $\mathbf{Z}_{\text {PPTE }}$ & 2,9 \\
\hline \multirow{4}{*}{ SPAR } & $\mathbf{X}_{\text {SPAR }}$ & 2,1 \\
\cline { 2 - 3 } & $\mathbf{Y}_{\text {SPAR }}$ & 2,1 \\
\cline { 2 - 3 } & $\mathbf{Z}_{\text {SPAR }}$ & 2,1 \\
\hline \multirow{4}{*}{ ILHA } & $\mathbf{X}_{\text {ILHA }}$ & 2,8 \\
\cline { 2 - 3 } & $\mathbf{Y}_{\text {ILHA }}$ & 2,8 \\
\cline { 2 - 3 } & $\mathbf{Z}_{\text {ILHA }}$ & 2,8 \\
\hline \multirow{5}{*}{ OURI } & $\mathbf{X}_{\text {OURI }}$ & 3,4 \\
\cline { 2 - 3 } & $\mathbf{Y}_{\text {OURI }}$ & 3,4 \\
\cline { 2 - 3 } & $\mathbf{Z}_{\text {OURI }}$ & 3,4 \\
\hline
\end{tabular}

Analisando os resultados apresentados na Tabela 4, nota-se que com estas doze linhas-base, o critério de planejamento da precisão da rede geodésica (coordenadas dos pontos com um desvio padrão de no máximo $5 \mathrm{~cm}$ ) continua sendo satisfeito. Analisado e comparando os resultados das Tabelas 2 e 4, nota-se ainda que a precisão esperada para os parâmetros melhorou (ou seja, o desvio padrão estimado para estes diminuiu), o que já se era esperado, pois foram adicionadas mais três linhas-base para a geometria/configuração da rede, aumentando assim o número de observações (e consequentemente a redundância do ajustamento). Finalmente, resta ainda verificar a confiabilidade da rede geodésica neste segundo cenário, com as três linhas-base adicionais (ILHA - SPAR, OURI - SPAR, SPAR - SJRP).

Mantendo o valor para o parâmetro de não centralidade do modelo em $\lambda_{0}=$ 17,075, resolveu-se novamente o problema de autovalores e autovetores da expressão (3) para estimar a influência máxima de possíveis erros não detectados em cada par de observações sobre cada parâmetro. Para este cenário, onde o número de observações se torna $\mathrm{n}=3 \times 12=36$ (componentes $\Delta \mathrm{X}, \Delta \mathrm{Y}$ e $\Delta \mathrm{Z}$ das doze linhas-base independentes), e o número de observações suspeitas (simultaneamente) consideradas é $\mathrm{q}=2$, tem-se $\left(\begin{array}{l}n \\ q\end{array}\right)=\left(\begin{array}{c}36 \\ 2\end{array}\right)=630$ confiabilidades externas possíveis para cada um dos 12 parâmetros incógnitos da rede. A Tabela 5 apresenta o par de observações que resulta na confiabilidade externa máxima sobre cada parâmetro, bem como os valores destas influências máximas em cada parâmetro. 
Tabela 5 - Pares de observações com confiabilidade externa máxima sobre cada parâmetro da rede (com as três linhas-base adicionais).

\begin{tabular}{|c|c|c|c|}
\hline Ponto & Coordenada & $\begin{array}{c}\text { Par de observações com } \\
\text { confiabilidade externa máxima }\end{array}$ & $\begin{array}{c}\text { Influência } \\
\text { máxima }\end{array}$ \\
\hline \multirow{3}{*}{ PPTE } & $\mathbf{X}_{\text {PPTE }}$ & $\Delta \mathrm{X}(\mathrm{PPT}$ - SPAR) e $\Delta \mathrm{X}(\mathrm{ROSA}-\mathrm{PPTE})$ & $15,2 \mathrm{~cm}$ \\
\hline & $\mathbf{Y}_{\text {PPTE }}$ & $\Delta \mathrm{Y}(\mathrm{PPT}$ - SPAR) e $\Delta \mathrm{Y}(\mathrm{ROSA}-\mathrm{PPTE})$ & $15,2 \mathrm{~cm}$ \\
\hline & $\mathbf{Z}_{\text {PPTE }}$ & $\Delta Z$ (PPT - SPAR) e $\Delta Z$ (ROSA - PPTE) & $15,2 \mathrm{~cm}$ \\
\hline \multirow{3}{*}{ SPAR } & $\mathbf{X}_{\text {SPAR }}$ & $\Delta X($ SJRP - SPAR) e $\Delta X($ SPAR - SJRP) & $13,8 \mathrm{~cm}$ \\
\hline & $\mathbf{Y}_{\text {SPAR }}$ & $\Delta \mathrm{Y}(\mathrm{SJRP}-\mathrm{SPAR})$ e $\Delta \mathrm{Y}(\mathrm{SPAR}-\mathrm{SJR})$ & $13,8 \mathrm{~cm}$ \\
\hline & $\mathbf{Z}_{\text {SPAR }}$ & $\Delta \mathrm{Z}(\mathrm{SJRP}$ - SPAR) e $\Delta \mathrm{Z}$ (SPAR - SJRP) & $13,8 \mathrm{~cm}$ \\
\hline \multirow{3}{*}{ ILHA } & $\mathbf{X}_{\text {ILHA }}$ & $\Delta \mathrm{X}(\mathrm{SPAR}$ - ILHA) e $\Delta \mathrm{X}$ (ILHA - SPAR) & $13,8 \mathrm{~cm}$ \\
\hline & $\mathbf{Y}_{\text {ILHA }}$ & $\Delta Y($ SPAR - ILHA) e $\Delta Y$ (ILHA - SPAR) & $13,8 \mathrm{~cm}$ \\
\hline & $\mathbf{Z}_{\text {ILHA }}$ & $\Delta \mathrm{Z}$ (SPAR - ILHA) e $\Delta \mathrm{Z}$ (ILHA - SPAR) & $13,8 \mathrm{~cm}$ \\
\hline \multirow{3}{*}{ OURI } & $\mathbf{X}_{\text {OURI }}$ & $\begin{array}{c}\Delta \mathrm{X}(\mathrm{PPTE}-\mathrm{OURI}) \text { e } \triangle \mathrm{X}(\mathrm{SPAR}-\mathrm{OURI} / \\
\text { OURI - SPAR) }\end{array}$ & $13,3 \mathrm{~cm}$ \\
\hline & $\mathbf{Y}_{\text {OURI }}$ & $\begin{array}{c}\Delta \mathrm{Y}(\mathrm{PPTE}-\mathrm{OURI}) \text { e } \Delta \mathrm{Y}(\mathrm{SPAR} \text { - OURI / } \\
\text { OURI - SPAR) }\end{array}$ & $13,3 \mathrm{~cm}$ \\
\hline & $\mathbf{Z}_{\text {OURI }}$ & $\begin{array}{c}\Delta \mathrm{Z} \text { (PPTE - OURI) e } \Delta \mathrm{Z} \text { (SPAR - OURI / } \\
\text { OURI - SPAR) }\end{array}$ & $13,3 \mathrm{~cm}$ \\
\hline
\end{tabular}

Analisando a Tabela 5, nota-se que nenhuma coordenada dos vértices SPAR, OURI e ILHA apresenta uma confiabilidade externa (máxima) superior a $15 \mathrm{~cm}$. Somente para o vértice PPTE, a influência máxima do par de linhas-base PPT SPAR e ROSA - PPTE supera em $2 \mathrm{~mm}$ o limite pré-estabelecido. Como estes valores são estimativas, sujeitos, portanto, a certa tolerância, optou-se por, ao invés de se adicionar mais uma linha-base à rede geodésica (aumentando assim os custos e o tempo de execução do projeto), considerar que com esta geometria/configuração, e com esta precisão esperada para as observações, os critérios de acurácia (precisão e confiabilidade) da rede GNSS seriam satisfeitos.

Portanto, a etapa de pré-análise (planejamento) da rede geodésica, pelo método da tentativa e erro, está concluída, ou seja, com estas doze linhas-base independentes, e com esta estratégia de ponderação para a precisão das observações (0,5 ppm), espera-se uma precisão para as coordenadas dos pontos da rede melhor do que $5 \mathrm{~cm}$, e uma influência máxima de possíveis erros (não detectados) em até duas observações de no máximo $15 \mathrm{~cm}$ para cada uma destas coordenadas.

Para verificar se de fato, na prática, estes critérios são satisfeitos, a próxima etapa do experimento é o processamento dos dados das estações (disponíveis gratuitamente pelo site do IBGE), bem como o ajustamento da rede GNSS, para comparar os resultados obtidos empiricamente com aqueles que foram estimados teoricamente na etapa de pré-análise. 
Desta forma, os dados utilizados para o processamento foram relativos ao mês de Janeiro do ano de 2010. Visando tornar o experimento mais realístico, considerando que houve a disponibilidade de apenas dois receptores GNSS de dupla frequência para o projeto, foram processadas duas linhas-base por dia (com tempos de rastreio de 6h cada), o que resultaria em uma etapa de campo de seis dias (pois são doze linhas-base independentes). Todos os dados das estações utilizados foram obtidos pelo site do IBGE, na seção de downloads da RBMC (disponível no seguinte endereço eletrônico: http://www.ibge.gov.br/home/geociencias/download/ tela_inicial.php?tipo $=8$ ).

Após realizar o processamento dos doze posicionamentos relativos (um para cada linha-base), foram obtidas as componentes $\Delta \mathrm{X}, \Delta \mathrm{Y}$ e $\Delta \mathrm{Z}$ de cada linha-base, bem como as respectivas variâncias e covariâncias destas componentes. A Tabela 6 apresenta os desvios-padrão das observações que foram estimados a priori (na etapa de planejamento da rede), e os desvios-padrão destas obtidos com o processamento dos dados.

Tabela 6 - Desvios-padrão preditos (no planejamento) e obtidos (no processamento dos dados) para cada componente $(\Delta \mathrm{X}, \Delta \mathrm{Y}$ e $\Delta \mathrm{Z})$ das doze linhas-base.

\begin{tabular}{c|c|c|c|c}
\hline Linha-base & $\boldsymbol{\sigma}$ - predito & $\boldsymbol{\sigma}_{\Delta \mathbf{X}}-$ obtido & $\boldsymbol{\sigma}_{\Delta \mathbf{Y}}-$ obtido & $\boldsymbol{\sigma}_{\Delta \mathbf{Z}}-$ obtido \\
\hline PPTE - SPAR & $0,042 \mathrm{~m}$ & $0,034 \mathrm{~m}$ & $0,039 \mathrm{~m}$ & $0,025 \mathrm{~m}$ \\
\hline PPTE - ILHA & $0,054 \mathrm{~m}$ & $0,041 \mathrm{~m}$ & $0,04 \mathrm{~m}$ & $0,031 \mathrm{~m}$ \\
\hline PPTE - OURI & $0,052 \mathrm{~m}$ & $0,039 \mathrm{~m}$ & $0,041 \mathrm{~m}$ & $0,032 \mathrm{~m}$ \\
\hline SJRP - SPAR & $0,035 \mathrm{~m}$ & $0,033 \mathrm{~m}$ & $0,033 \mathrm{~m}$ & $0,025 \mathrm{~m}$ \\
\hline SJRP - ILHA & $0,061 \mathrm{~m}$ & $0,06 \mathrm{~m}$ & $0,037 \mathrm{~m}$ & $0,023 \mathrm{~m}$ \\
\hline SJRP - OURI & $0,071 \mathrm{~m}$ & $0,04 \mathrm{~m}$ & $0,048 \mathrm{~m}$ & $0,041 \mathrm{~m}$ \\
\hline SPAR - ILHA & $0,036 \mathrm{~m}$ & $0,032 \mathrm{~m}$ & $0,035 \mathrm{~m}$ & $0,025 \mathrm{~m}$ \\
\hline SPAR - OURI & $0,059 \mathrm{~m}$ & $0,042 \mathrm{~m}$ & $0,044 \mathrm{~m}$ & $0,032 \mathrm{~m}$ \\
\hline ROSA - PPTE & $0,048 \mathrm{~m}$ & $0,036 \mathrm{~m}$ & $0,041 \mathrm{~m}$ & $0,027 \mathrm{~m}$ \\
\hline ILHA - SPAR & $0,036 \mathrm{~m}$ & $0,035 \mathrm{~m}$ & $0,034 \mathrm{~m}$ & $0,022 \mathrm{~m}$ \\
\hline OURI - SPAR & $0,059 \mathrm{~m}$ & $0,057 \mathrm{~m}$ & $0,036 \mathrm{~m}$ & $0,022 \mathrm{~m}$ \\
\hline SPAR - SJRP & $0,035 \mathrm{~m}$ & $0,031 \mathrm{~m}$ & $0,037 \mathrm{~m}$ & $0,021 \mathrm{~m}$ \\
\hline
\end{tabular}

Analisando a Tabela 6, nota-se que em praticamente todos os casos, o desviopadrão das componentes das linhas-base foram menores (ou no máximo iguais) do que os desvios-padrão estimados na etapa de pré-análise. Somente para a componente $\Delta \mathrm{Y}$ da linha-base SPAR - SJRP, o desvio-padrão foi $2 \mathrm{~mm}$ maior do que o valor estimado a priori. Ou seja, esta estratégia de ponderação para a precisão das linhas-base (de $0,5 \mathrm{ppm}$ ) se mostrou coerente com os resultados obtidos com o processamento dos dados. Ainda analisando a Tabela 7, nota-se que as componentes 
$\Delta \mathrm{Z}$ das doze linhas-base apresentam uma precisão melhor do que as componentes $\Delta \mathrm{X}$ e, principalmente, $\Delta \mathrm{Y}$, provavelmente devido ao fato das componentes $\Delta \mathrm{X}$ e $\Delta \mathrm{Y}$ serem maiores do que as componentes $\Delta \mathrm{Z}$, devido à localização geográfica da rede geodésica em estudo (de maior extensão na direção Leste - Oeste do que na direção Norte - Sul).

Com esta nova matriz peso das observações, obtida com os resultados do processamento dos dados, por meio da expressão (1), pode-se estimar a matriz de covariância para os parâmetros. Com as variâncias e covariâncias estimadas para os parâmetros desta maneira, pode-se então obter os desvios-padrões para os parâmetros, conforme mostra a Tabela 7 :

Tabela 7 - Precisão esperada e precisão obtida para cada coordenada dos quatro vértices desconhecidos da rede GNSS.

\begin{tabular}{c|c|c|c}
\hline Vértice & Parâmetro (coordenada) & Precisão esperada & Precisão obtida \\
\hline \multirow{4}{*}{ PPTE } & $\mathbf{X}_{\text {PPTE }}$ & $2,9 \mathrm{~cm}$ & $2,2 \mathrm{~cm}$ \\
\cline { 2 - 4 } & $\mathbf{Y}_{\text {PPTE }}$ & $2,9 \mathrm{~cm}$ & $2,4 \mathrm{~cm}$ \\
\cline { 2 - 4 } & $\mathbf{Z}_{\text {PPTE }}$ & $2,9 \mathrm{~cm}$ & $1,6 \mathrm{~cm}$ \\
\hline \multirow{3}{*}{ SPAR } & $\mathbf{X}_{\text {SPAR }}$ & $2,1 \mathrm{~cm}$ & $1,7 \mathrm{~cm}$ \\
\cline { 2 - 4 } & $\mathbf{Y}_{\text {SPAR }}$ & $2,1 \mathrm{~cm}$ & $1,8 \mathrm{~cm}$ \\
\cline { 2 - 4 } & $\mathbf{Z}_{\text {SPAR }}$ & $2,1 \mathrm{~cm}$ & $1,2 \mathrm{~cm}$ \\
\hline \multirow{4}{*}{ ILHA } & $\mathbf{X}_{\text {ILHA }}$ & $2,8 \mathrm{~cm}$ & $2,3 \mathrm{~cm}$ \\
\cline { 2 - 4 } & $\mathbf{Y}_{\text {ILHA }}$ & $2,8 \mathrm{~cm}$ & $2,2 \mathrm{~cm}$ \\
\hline \multirow{3}{*}{ OURI } & $\mathbf{Z}_{\text {ILHA }}$ & $2,8 \mathrm{~cm}$ & $1,5 \mathrm{~cm}$ \\
\cline { 2 - 4 } & $\mathbf{X}_{\text {OURI }}$ & $3,4 \mathrm{~cm}$ & $2,3 \mathrm{~cm}$ \\
\cline { 2 - 4 } & $\mathbf{Y}_{\text {OURI }}$ & $3,4 \mathrm{~cm}$ & $2,5 \mathrm{~cm}$ \\
\hline
\end{tabular}

Analisando a Tabela 7, nota-se que de fato, a precisão obtida para os parâmetros, com o processamento dos dados, foi melhor do que $5 \mathrm{~cm}$ (sendo em todos os casos, melhor do que precisão esperada na etapa de pré-análise). Nota-se também, que a precisão da coordenada cartesiana geocêntrica $Z$ dos vértices é ligeiramente melhor do que a precisão das coordenadas cartesianas geocêntricas $\mathrm{X}$ e $\mathrm{Y}$, devido ao fato já comentado das componentes $\Delta \mathrm{Z}$ apresentarem um desviopadrão menor do que as componentes $\Delta \mathrm{X}$ e $\Delta \mathrm{Y}$ por causa da localização geográfica da rede geodésica em estudo.

Por fim, com os resultados obtidos com o processamento das doze linhas-base (considerando as variâncias e covariâncias de suas componentes), resolveu-se o problema de autovalores e autovetores da expressão (3) para estimar a influência máxima de possíveis erros não detectados em cada par de observações sobre cada 
parâmetro. A Tabela 8 apresenta o par de observações que resultou na confiabilidade externa máxima sobre cada parâmetro, bem como os valores destas influências máximas em cada parâmetro.

Tabela 8 - Pares de observações com confiabilidade externa máxima sobre cada parâmetro da rede (após o processamento dos dados GNSS).

\begin{tabular}{|c|c|c|c|}
\hline Vértice & Coordenada & $\begin{array}{c}\text { Par de observações com } \\
\text { confiabilidade externa máxima }\end{array}$ & $\begin{array}{c}\text { Influência } \\
\text { maxima }\end{array}$ \\
\hline \multirow{3}{*}{ PPTE } & $\mathbf{X}_{\text {PPTE }}$ & $\Delta \mathrm{X}(\mathrm{PPT}-\mathrm{SPAR})$ e $\Delta \mathrm{X}(\mathrm{ROSA}-\mathrm{PPTE})$ & $7,4 \mathrm{~cm}$ \\
\hline & $\mathbf{Y}_{\text {PPTE }}$ & $\Delta \mathrm{Y}(\mathrm{PPT}-\mathrm{ILHA})$ e $\Delta \mathrm{Y}(\mathrm{ROSA}-\mathrm{PPTE})$ & $6,7 \mathrm{~cm}$ \\
\hline & $\mathbf{Z}_{\text {PPTE }}$ & $\Delta \mathrm{Z}(\mathrm{PPT}-\mathrm{SPAR})$ e $\Delta \mathrm{Z}(\mathrm{ROSA}-\mathrm{PPTE})$ & $6,3 \mathrm{~cm}$ \\
\hline \multirow{3}{*}{ SPAR } & $\mathbf{X}_{\text {SPAR }}$ & $\Delta \mathrm{X}(\mathrm{SJRP}-\mathrm{SPAR})$ e $\Delta \mathrm{X}(\mathrm{SPAR}-\mathrm{SJRP})$ & $7,1 \mathrm{~cm}$ \\
\hline & $\mathbf{Y}_{\text {SPAR }}$ & $\Delta \mathrm{Y}(\mathrm{SJRP}-\mathrm{SPAR})$ e $\Delta \mathrm{Y}(\mathrm{SPAR}-\mathrm{SJR})$ & $6,1 \mathrm{~cm}$ \\
\hline & $\mathbf{Z}_{\text {SPAR }}$ & $\Delta \mathrm{Z}(\mathrm{SJRP}-\mathrm{SPAR})$ e $\Delta \mathrm{Z}(\mathrm{SPAR}-\mathrm{SJRP})$ & $4,9 \mathrm{~cm}$ \\
\hline \multirow{3}{*}{ ILHA } & $\mathbf{X}_{\text {ILHA }}$ & $\begin{array}{c}\Delta \mathrm{X}(\mathrm{SPAR}-\mathrm{ILHA}) \text { e } \Delta \mathrm{X}(\mathrm{ILHA}- \\
\text { SPAR })\end{array}$ & $8,5 \mathrm{~cm}$ \\
\hline & $Y_{\text {ILHA }}$ & $\Delta \mathrm{Y}(\mathrm{SJRP}-\mathrm{SPAR})$ e $\Delta \mathrm{Y}(\mathrm{SJRP}-\mathrm{ILHA})$ & $6,4 \mathrm{~cm}$ \\
\hline & $\mathbf{Z}_{\text {ILHA }}$ & $\Delta \mathrm{Z}(\mathrm{SJRP}-\mathrm{ILHA})$ e $\Delta \mathrm{Z}$ (ILHA - SPAR) & $5,7 \mathrm{~cm}$ \\
\hline \multirow{3}{*}{ OURI } & $\mathbf{X}_{\text {OURI }}$ & $\Delta \mathrm{X}(\mathrm{PPTE}-\mathrm{OURI})$ e $\Delta \mathrm{X}(\mathrm{SJRP}-\mathrm{OURI})$ & $8,8 \mathrm{~cm}$ \\
\hline & $\mathbf{Y}_{\text {OURI }}$ & $\Delta \mathrm{Y}(\mathrm{SJRP}-\mathrm{OURI})$ e $\Delta \mathrm{Y}(\mathrm{OURI}-\mathrm{SPAR})$ & $6,3 \mathrm{~cm}$ \\
\hline & $\mathbf{Z}_{\text {OURI }}$ & $\begin{array}{c}\Delta \mathrm{Z}(\mathrm{SPAR}-\mathrm{OURI}) \text { e } \Delta \mathrm{Z}(\mathrm{OURI}- \\
\text { SPAR })\end{array}$ & $6,6 \mathrm{~cm}$ \\
\hline
\end{tabular}

Analisando a Tabela 8 , nota-se que nenhuma coordenada dos quatro vértices desconhecidos apresenta uma confiabilidade externa (máxima) superior a $15 \mathrm{~cm}$. Mais do que isso, estas confiabilidades externas máximas foram inferiores a $10 \mathrm{~cm}$, com uma influência máxima de $8,8 \mathrm{~cm}$ das componentes $\Delta \mathrm{X}$ (PPTE - OURI) e $\Delta \mathrm{X}$ (SJRP - OURI) sobre a coordenada X da estação OURI. Ou seja, de fato os critérios de acurácia (precisão e confiabilidade) que foram pré-estabelecidos, foram satisfeitos com esta geometria/configuração da rede geodésica, com doze linhasbase independentes. Além disso, analisando e comparando as Tabelas 5 e 8, nota-se também que os pares de linha-base que apresentaram maiores influências sobre os parâmetros não foram iguais aos previstos na etapa de pré-análise em todos os $(\mathrm{u}=$ 12) casos, pois embora a matriz design A fosse mantida a mesma, a matriz de covariância das observações obtida com o processamento dos dados foi diferente da matriz de covariância que foi estimada na etapa de pré-análise (além das variâncias "esperadas" e "obtidas" serem relativamente diferentes, com o processamento dos dados foi possível utilizar também as covariâncias entre as componentes de cada linha-base na matriz de covariância das observações). 
Dando continuidade aos experimentos, resta ainda verificar a possível existência de erros (não aleatórios) nas observações ou no modelo matemático. Para o controle de qualidade da rede, foi aplicado o teste global do ajustamento para a detecção de erros nas observações e/ou no modelo, e o procedimento data snooping para a identificação das observações suspeitas de estarem contaminadas. Para o teste global do ajustamento (BAARDA, 1968; KAVOURAS, 1982; TEUNISSEN, 2006; KLEIN, 2012), a estatística de teste resultou em $\mathrm{T}_{\mathrm{q}}=25,86$ (e o fator de variância $a$ posteriori resultou em $\left.\widehat{\sigma}^{2}=1,08\right)$. O valor crítico para o teste, tabelado na distribuição qui quadrado em função do número de graus de liberdade do ajustamento $(\mathrm{q}=\mathrm{n}-\mathrm{u}=36-12=24)$, e de um nível de significância $\alpha=0,036$, é $\chi_{\alpha}^{2}=37,84$. Portanto, como a estatística calculada é menor do que o valor crítico tabelado, não há evidências da existência de erros no modelo e/ou nas observações, ao nível de significância $\alpha=0,036$.

O valor para o nível de significância do teste global foi obtido em função do nível de significância adotado para o procedimento data snooping $\left(\alpha_{0}=0,001\right)$, por meio da desigualdade de Bonferroni (KAVOURAS, 1982; KLEIN, 2012): $\alpha=\mathrm{n} \alpha_{0}$. Esta dependência entre o nível de significância do teste global e do procedimento data snooping visa garantir o mesmo poder de teste para ambos (que neste caso, foi estipulado em $\gamma=0,8$ ), pois o número de graus de liberdade destes dois testes é diferente (para maiores detalhes ver BAARDA, 1968; KAVOURAS, 1982; TEUNISSEN, 2006; KLEIN, 2012).

Seguindo a recomendação de Kavouras (1982), mesmo que o teste global do ajustamento tenha sido "não rejeitado", ainda assim foi aplicado o procedimento data snooping para a identificação de observações suspeitas de estarem contaminadas por erros. Neste caso, foi utilizada a estatística de teste $\mathrm{T}_{\mathrm{q}}$ definida em (6), com a matriz $C_{L}$ se tornando o vetor unitário $c_{l i}$ correspondente a linha da iésima observação testada (ver, por exemplo, BAARDA, 1968 e KLEIN et al., 2011). Aplicando o procedimento data snooping para todas as $(\mathrm{n}=36)$ observações, a que apresentou a maior estatística de teste foi a observação $\Delta \mathrm{Y}$ da linha-base ROSA-PPTE, com o valor de $\mathrm{T}_{\mathrm{q}}=4,41$. $\mathrm{O}$ valor crítico tabelado para a distribuição qui quadrado, com q $=1$ grau de liberdade (data snooping) e nível de significância $\alpha_{0}=0,001$, é $\chi_{\alpha}^{2}=10,83$. Portanto, como nenhuma observação apresentou uma estatística de teste $\left(\mathrm{T}_{\mathrm{q}}\right)$ maior do que o valor crítico tabelado $\left(\chi_{\alpha}^{2}\right)$, nenhuma observação pode ser considerada suspeita de estar contaminada por erros, ao nível de significância $\alpha_{0}=0,001$.

Mesmo que o procedimento data snooping não tenha identificado nenhuma observação contaminada por erros (ao nível de significância $\alpha_{0}=0,001$ ), também foi aplicado o teste para identificação de erros com $q=2$ (ou seja, considerando duas observações suspeitas, simultaneamente, ao contrário do data snooping, onde apenas $q=1$ observação é considerada suspeita por vez), uma vez que estes testes para identificação de erros podem ser suscetíveis a algumas armadilhas, como por exemplo, os fenômenos swamping e masking (GUI et al., 2010; KLEIN, 2012). Para 
estes testes, o valor crítico tabelado para a distribuição qui quadrado, com $\mathrm{q}=2$ graus de liberdade e nível de significância $\alpha=0,0027$, é dado por $\chi_{\alpha}^{2}=11,83$. Novamente, o nível de significância foi obtido em função do nível de significância do data snooping, pois o parâmetro de não centralidade do modelo $\left(\lambda_{0}\right)$, utilizado para calcular as medidas de confiabilidade externa por meio da expressão (3), também foi determinado com base neste valor para o nível de significância ( $\alpha=$ 0,0027 ), sendo que a relação entre o nível de significância do teste para $q=2$ e do procedimento data snooping (onde $\mathrm{q}=1$ ), foi obtida em um monograma em Baarda (1968). Ressalva-se que o poder do teste também foi considerado o mesmo em ambos os casos (ou seja, $\gamma=0,8$ ). Uma discussão mais detalhada sobre a obtenção do parâmetro $\lambda_{0}$ para testes estatísticos com diferentes valores para $\alpha, \gamma$ e q pode ser obtida em Baarda (1968), Kavouras (1982), Teunissen (2006) e Klein (2012).

Dentre todas as $\left(\begin{array}{l}n \\ q\end{array}\right)=\left(\begin{array}{c}36 \\ 2\end{array}\right)=630$ combinações possíveis para $q=2$ (ou seja, duas observações consideradas suspeitas por vez), a que apresentou a maior estatística de teste foi o par de observações $\Delta \mathrm{Y}$ (PPTE - OURI) e $\Delta Y$ (ILHA SPAR), com $\mathrm{T}_{\mathrm{q}}=6,59$. O valor crítico tabelado para a distribuição qui quadrado, com $q=2$ graus de liberdade e nível de significância $\alpha_{0}=0,0027$, é $\chi_{\alpha}^{2}=11,83$. Ou seja, nenhum par de observações pode ser considerado, simultaneamente, contaminado por erros, ao nível de significância $\alpha_{0}=0,0027$ (pois 6,59<11,83).

Por fim, como a confiabilidade da rede geodésica foi planejada neste cenário com dois outliers simultâneos (não detectados), erros propositais foram inseridos em duas observações para verificar se a influência destes sobre os parâmetros está de acordo com os valores de confiabilidade externa que foram obtidos. Desta forma, o par de observações escolhido neste último experimento foram as observações $\Delta \mathrm{Z}$ da linha-base SJRP - ILHA e $\Delta \mathrm{Z}$ da linha-base SPAR - ILHA.

A Tabela 9 apresenta os erros inseridos nas duas observações e o resultado do teste definido em (4), para $q=2$, com estas duas observações sendo consideradas, simultaneamente, suspeitas de estarem contaminadas por erros.

Tabela 9 - Erros propositais inseridos nas observações $\Delta Z$ (SJRP - ILHA) e $\Delta Z$ (SPAR - ILHA) e o resultado do teste para identificação de erros simultâneos nestas duas observações.

\begin{tabular}{|c|c|c|}
\hline Observações & Erros Inseridos & Resultado do teste para $q=2$ \\
\hline $\begin{array}{l}\Delta \mathrm{Z} \text { (SJRP - ILHA) e } \\
\Delta \mathrm{Z} \text { (SPAR - ILHA) }\end{array}$ & $+5 \mathrm{~cm} \mathrm{em} \mathrm{cada}$ & $\mathrm{H}_{0}$ não rejeitada (erros não detectados) \\
\hline $\begin{array}{c}\Delta \mathrm{Z}(\mathrm{SJRP}-\mathrm{ILHA}) \mathrm{e} \\
\Delta \mathrm{Z}(\mathrm{SPAR}-\mathrm{ILHA})\end{array}$ & $+10 \mathrm{~cm}$ em cada & $\mathrm{H}_{0}$ rejeitada (erros detectados) \\
\hline
\end{tabular}

Novamente para estes testes, o valor crítico tabelado para a distribuição qui quadrado, com q $=2$ graus de liberdade e nível de significância $\alpha=0,0027$, é $\chi_{\alpha}^{2}=$ 11,83. Analisando a Tabela 9 , nota-se que erros de $+10 \mathrm{~cm}$ nas observações $\Delta Z$ 
(SJRP - ILHA) e $\Delta Z$ (SPAR - ILHA) são detectados (simultaneamente) por meio da aplicação do teste para $\mathrm{q}=2$, enquanto erros de $+5 \mathrm{~cm}$ nestas observações não são detectados (simultaneamente) por meio deste teste. Como o objetivo é verificar a influência máxima de possíveis erros não detectados nestas duas observações, foram inseridos erros de magnitude entre $+5 \mathrm{~cm} \mathrm{e}+10 \mathrm{~cm}$, e os resultados do teste são apresentados na Tabela 10 .

Tabela 10 - Erros propositais inseridos nas observações $\Delta Z$ (SJRP - ILHA) e $\Delta Z$ (SPAR - ILHA) e o resultado do teste para identificação de erros simultâneos nestas

\begin{tabular}{c|c|c}
\multicolumn{3}{c}{ duas observações. } \\
\hline Observações & Erros Inseridos & Resultado do teste para q = 2 \\
\hline$\Delta$ Z (SJRP - ILHA) e & $+6 \mathrm{~cm}$ em cada & $\mathrm{H}_{0}$ rejeitada (erros detectados) \\
$\Delta \mathrm{Z}$ (SPAR - ILHA) & + . \\
\hline
\end{tabular}

Analisando as Tabelas 9 e 10, nota-se que a magnitude máxima de erros não detectados nestas duas observações (de maneira simultânea), é de cerca de $6 \mathrm{~cm}$. Ou seja, erros desta magnitude podem ser interpretados, de maneira aproximada, como os "menores erros detectáveis" nestas observações, ou analogamente, como os "maiores erros nãos detectáveis" nestas observações (de maneira simultânea). Assim sendo, mantendo estes erros propositais de $+6 \mathrm{~cm}$ nestas duas observações, determinou-se o valor das coordenadas $Z$ dos vértices desconhecidos PPTE, SPAR, ILHA e OURI, por meio do ajustamento da rede, e se comparou estes resultados com os valores obtidos para estas coordenadas pelo ajustamento da rede, mas sem os erros propositais nestas observações, sendo que ambos os resultados obtidos são apresentados na Tabela 11 .

Tabela 11 - Valores estimados para a coordenada $Z$ dos vértices da rede (ajustamento sem erros propositais em $\Delta Z$ (SJRP - ILHA) e $\Delta$ Z (SPAR - ILHA), e ajustamento com erros propositais nestas duas observações).

\begin{tabular}{c|c|c}
\hline Parâmetro & $\begin{array}{c}\text { Valor estimado - } \\
\text { Ajustamento sem } \\
\text { erros inseridos } \\
\text { nas observações }\end{array}$ & $\begin{array}{c}\text { Valor estimado - Ajustamento } \\
\text { com erros de +6 cm nas observações } \\
\Delta \text { Z (SJRP - ILHA) e } \Delta \text { Z (SPAR - ILHA) }\end{array}$ \\
\hline$Z_{\text {PPTE }}$ & $-2386880,414 \mathrm{~m}$ & $-2386880,403 \mathrm{~m}$ \\
\hline$Z_{\text {SPAR }}$ & $-2290619,399 \mathrm{~m}$ & $-2290619,381 \mathrm{~m}$ \\
\hline$Z_{\mathrm{ILHA}}$ & $-2212269,499 \mathrm{~m}$ & $-2212269,488 \mathrm{~m}$ \\
\hline$Z_{\mathrm{OURI}}$ & $-2471710,683 \mathrm{~m}$ & $-2471710,668 \mathrm{~m}$ \\
\hline
\end{tabular}

Analisando a Tabela 11, nota-se que a diferença nos valores da coordenada $\mathrm{Z}$ dos vértices desconhecidos, realizando o ajustamento da rede com as observações originais, e realizando o ajustamento da rede com erros propositais de $+6 \mathrm{~cm} \mathrm{em}$ 
$\Delta \mathrm{Z}$ (SJRP - ILHA) e $\Delta \mathrm{Z}$ (SPAR - ILHA), situam-se entre $1,2 \mathrm{~cm}$ e $1,9 \mathrm{~cm}$. Para verificar se os valores de confiabilidade externa máxima calculados foram de fato concordantes com esta realidade, estas diferenças são comparadas com a influência máxima estimada destas observações sobre estes parâmetros, conforme mostra a Tabela 12.

Tabela 12 - Influência de erros (propositais) "não detectados" em $\Delta \mathrm{Z}$ (SJRP ILHA) e $\Delta Z$ (SPAR - ILHA) e a influência máxima estimada (confiabilidade externa) para erros não detectados neste par de observações.

\begin{tabular}{|c|c|c|}
\hline Parâmetro & $\begin{array}{c}\text { Influência de erros } \\
\quad \text { (propositais) } \\
\text { “não detectados" em } \\
\Delta \mathrm{Z} \text { (SJRP - ILHA) e } \\
\Delta \mathrm{Z} \text { (SPAR - ILHA) } \\
\end{array}$ & $\begin{array}{c}\text { Influência máxima estimada } \\
\text { para erros não detectados em } \\
\Delta \mathrm{Z} \text { (SJRP - ILHA) e } \\
\Delta \mathrm{Z} \text { (SPAR - ILHA) }\end{array}$ \\
\hline$Z_{\text {PPTE }}$ & $1,1 \mathrm{~cm}$ & $1,9 \mathrm{~cm}$ \\
\hline$Z_{\text {SPAR }}$ & $1,8 \mathrm{~cm}$ & $1,9 \mathrm{~cm}$ \\
\hline $\mathrm{Z}_{\mathrm{ILHA}}$ & $1,1 \mathrm{~cm}$ & $5,3 \mathrm{~cm}$ \\
\hline $\mathrm{Z}_{\text {OURI }}$ & $1,5 \mathrm{~cm}$ & $1,6 \mathrm{~cm}$ \\
\hline
\end{tabular}

Analisando a Tabela 12, nota-se que, de fato, a confiabilidade externa máxima das observações sobre os parâmetros, se mostrou concordante com a realidade, pois, empiricamente, se constatou que erros de cerca de $6 \mathrm{~cm}$ são os erros de magnitude máxima "não detectados", simultaneamente, nas observações $\Delta \mathrm{Z}$ (SJRP - ILHA) e $\triangle \mathrm{Z}$ (SPAR - ILHA), e a influência destes erros sobre os parâmetros (coordenadas Z dos vértices desconhecidos PPTE, SPAR, ILHA e OURI), é menor do que a influência máxima destas observações sobre estes parâmetros, obtida por meio da confiabilidade externa para este par de observações, por meio da expressão (3). Nota-se também a alta concordância para os casos da coordenada $Z$ dos vértices SPAR e OURI, embora o verdadeiro poder do teste, que foi arbitrado em $80 \%$ para obtenção do parâmetro de não centralidade do modelo $\left(\lambda_{0}\right)$, seja desconhecido na prática. Para a coordenada $Z$ da estação ILHA, a influência obtida $(1,1 \mathrm{~cm})$ foi bem menor do que a influência máxima prevista $(5,3 \mathrm{~cm})$, o que mostra que estes valores de confiabilidade externa para múltiplos outliers não superestimam a qualidade das observações, sendo concordantes com a realidade, ou até mesmo, superestimando a influência de possíveis erros não detectados nas observações sobre os parâmetros.

Assim sendo, pode-se dizer que as coordenadas dos quatro vértices incógnitos desta rede GNSS (PPTE, SPAR, ILHA e OURI), obtidas por meio do ajustamento de doze linhas-base independentes (com tempo de rastreio de $6 \mathrm{~h}$ cada), apresentam um desvio-padrão de no máximo $2,5 \mathrm{~cm}$, e, além disso, a influência máxima de possíveis erros não detectados em até duas observações (de maneira simultânea), 
sobre estas coordenadas, é inferior à $10 \mathrm{~cm}$, satisfazendo os critérios de acurácia que foram pré-estabelecidos (estipulados) para esta rede geodésica.

\section{CONCLUSÃO}

Quando se deseja planejar uma rede geodésica visando atender critérios de confiabilidade, se utiliza da teoria convencional de confiabilidade, que considera somente a existência de uma observação suspeita de estar contaminada por erros (não aleatórios) por vez. Recentemente, a teoria convencional de confiabilidade foi ampliada (generalizada) para o caso em que o número de observações suspeitas consideradas é maior do que um. Neste sentido, este artigo teve como principal objetivo apresentar um exemplo do planejamento de uma rede geodésica baseado na teoria de confiabilidade generalizada. Apresentou-se uma revisão geral sobre planejamento de redes e sobre a teoria de confiabilidade generalizada, e se exemplificou o planejamento de uma rede GNSS, seguindo a revisão apresentada.

No exemplo realizado, se buscou realizar o planejamento de uma rede GNSS com quatro vértices incógnitos, de modo que a precisão das coordenadas ajustadas dos vértices fosse de $5 \mathrm{~cm}$, e a influência máxima de possíveis erros não detectados em até duas observações, sobre estas coordenadas (de maneira simultânea), não fosse maior do que $15 \mathrm{~cm}$.

Por meio de alterações na geometria/configuração da rede (número de linhasbase), e consequentemente na matriz design A do ajustamento, foi possível atender a estes critérios na etapa de pré-análise, por meio do método da tentativa e erro. Salienta-se que também poderiam ser alteradas as precisões assumidas para as observações, e/ou os níveis de probabilidade assumidos para os testes de identificação de erros, ficando estes critérios de acordo com os objetivos do projeto e as preferências do geodesista que estiver planejando a rede. De qualquer forma, recomenda-se o uso somente da confiabilidade externa máxima para múltiplos outliers (simultâneos), em virtude do grande número de cálculos envolvidos nas medidas de confiabilidade generalizadas para múltiplos outliers, e pelo fato de que na prática, se está mais interessado na influência de possíveis erros não detectados nas observações sobre os parâmetros (confiabilidade externa), do que na magnitude destes erros observacionais não detectados (confiabilidade interna).

Para validar os resultados obtidos na etapa de pré-análise, foram processadas as doze linhas-base da rede GNSS proposta, com tempo de ocupação de 6h cada, e posteriormente, foi realizado o ajustamento desta rede. Como resultados, obteve-se uma precisão para as coordenadas dos pontos de $2,5 \mathrm{~cm}$, e uma influência máxima de possíveis erros não detectados em duas observações sobre uma coordenada ajustada de $8,8 \mathrm{~cm}$, ou seja, os critérios de qualidade (precisão e confiabilidade) que foram pré-estabelecidos para a rede foram satisfeitos com a geometria/configuração que foi estabelecida para a mesma na etapa de pré-análise. Além disso, foram inseridos erros propositais em duas observações, de magnitude variável, até que estes erros não fossem detectados pelo teste para identificação de erros correspondente. Desta forma, comparando os valores das coordenadas ajustadas dos 
pontos (sem os erros propositais), com os valores destas coordenadas ajustadas (com os erros propositais nas duas observações testadas), verificou-se que estas discrepâncias eram menores ou iguais aos valores de confiabilidade externa máxima obtidos para este par de observações, ou seja, de fato estas medidas de confiabilidade resultam em valores passíveis de ocorrerem na prática, embora $\mathrm{o}$ verdadeiro poder do teste seja desconhecido.

Como recomendações para trabalhos futuros, pode-se citar estudos relativos ao planejamento de outros tipos de redes geodésicas (poligonais topográficas, aerotriangulações, redes de nivelamento geométrico e etc.), bem como um número maior de outliers simultâneos considerados, ou ainda, de redes GNSS cuja análise não seja feita em coordenadas cartesianas geocêntricas, mas em um sistema geodésico local (possibilitando fazer análises de planimetria e altimetria separadamente). Também se recomendam estudos, por meio do método da tentativa e erro, que não alterem somente a geometria/configuração da rede, como também a precisão esperada para as observações, ou até mesmo os níveis de probabilidade assumidos para o teste de identificação de erros.

\section{AGRADECIMENTOS:}

Os autores agradecem à FAPERGS/CAPES pelo fornecimento da bolsa de Doutorado do primeiro autor, ao CNPq pela Bolsa de Produtividade em Pesquisa (Proc.n.307472/2009-4) do segundo autor e à FAPERGS pela bolsa de Iniciação Cientifica da quarta autora.

\section{REFERÊNCIAS BIBLIOGRÁFICAS}

ALMAGBILE, A.; WANG, J.; DING, W.; KNIGHT, N. Sensitivity analysis of multiple fault test and reliability measures in integrated GPS/INS systems. In: 7th International Symposium on Mobile Mapping Technology, Cracow, Poland, 2011.

AMIRI-SIMKOOEI, A. M.; ASGARI, J.; ZANGENEH-NEJAD, F.; ZAMINPARDAZ, S. Basic concepts of optimization and design of geodetic networks. Journal of Surveying Engineering. Submitted June 22, 2011; accepted January 30, 2012; posted ahead of print February 1, 2012. doi:10.1061/(ASCE)SU.1943-5428.0000081.

AMORIM, G. P. Confiabilidade de rede GPS de referência cadastral municipal estudo de caso: rede do município de Vitória (ES). 2004. Dissertação (Mestrado em Transportes) - Escola de Engenharia de São Carlos, Universidade de São Paulo, São Carlos, 2004.

ALTAMIMI, Z.; COLLILIEUX, X.; METIVIER, L. ITRF2008: An improved solution of the International Terrestrial Reference Frame. Journal of Geodesy, 85(8), 457-473, 2011.

ANGUS, J. E. RAIM with multiple faults. Navigation, v. 53, n. 4, p. 249-257, 2006. 
BAARDA, W. A testing procedure for use in geodetic networks. Publications on Geodesy, New Series, v. 2, n. 5, Delft: Netherlands Geodetic Commission, 1968.

BAARDA, W. Measures for the accuracy of geodetic networks. In: Symposium on Optimization of Design and Computation of Control Networks, p. 419-436, Sopron, Hungary, 1977.

CHAVES, J. C. Uso da tecnologia GPS na monitoração de deformação: sistemas, etapas e experimentos. 2001. Tese (Doutorado em Transportes) - Escola de Engenharia de São Carlos, Universidade de São Paulo, São Carlos, 2001.

DALMOLIN, Q. Ajustamento por Mínimos Quadrados. Curitiba: Curso de PósGraduação em Ciências Geodésicas/UFPR, 2002. 175p.

DALMOLIN, Q.; OLIVEIRA, R. A Otimização dos Pesos das Observações Geodésicas por um Problema de Valor Próprio Inverso: Solução pelo Método de Newton e Quase-Newton - BFGS. Boletim de Ciências Geodésicas (Impresso), v. 16 - 4, p. 538-556, 2010.

FÖRSTNER, W. Reliability analysis of parameter estimation in linear models with applications to mensuration problems in computer vision. Computer Vision, Graphics and Image Processing, v. 40, p. 273-310, 1987.

GEMAEL, C. Introdução ao ajustamento de observações: aplicações geodésicas. Curitiba: Ed. UFPR, 1994. 319p.

GHILANI, C. D.; WOLF, P. R. Adjustment Computations: Spatial Data Analysis. 4. ed. New York: John Wiley \& Sons, 2006. 611p.

GRAFAREND, E. W. Optimization of geodetic networks. Boll. Geod. Sci. Aff. 33(4), 351-406, 1974.

GRAFAREND, E. W.; SANSO, F. Optimization and design of geodetic networks. Edited by Grafarend and Sanso, Springer-Verlag, Berlin, Heidelberg, New York, Tokyo, 1985.

GUI, Q.; LI, X.; GONG, Y.; LI, B.; LI, G. A Bayesian unmasking method for locating multiple gross errors based on posterior probabilities of classification variables. Journal of Geodesy, v. 85, n. 4, p. 191-203, 2010.

IBGE. Recomendações para Levantamentos Relativos Estáticos - GPS. 2008. Disponível em: ftp://geoftp.ibge.gov.br/documentos/geodesia/pdf/recom_gps_ internet.pdf. Acesso em: 03/04/2012.

KUANG, S. L. Geodetic network analysis and optimal design: concepts and application. Ann Arbor Press, Chelsea, MI, 1996.

KAVOURAS, M. On the Detection of Outliers and the Determination of Reliability in Geodetic Networks. 1982. M.Sc.E. Thesis - Department of Geodesy and Geomatics Engineering, University of New Brunswick, Fredericton, Canada, 1982.

KLEIN, I. Controle de Qualidade no Ajustamento de Observações Geodésicas. Dissertação (Mestrado) - Programa de Pós-Graduação em Sensoriamento Remoto, UFRGS. 2012. 
KLEIN, I.; MATSUOKA, M. T.; SOUZA, S. F. Teoria de Confiabilidade Generalizada para Múltiplos Outliers: Apresentação, Discussão e Comparação com a Teoria Convencional. Boletim de Ciências Geodésicas, v. 17, n. 4, p. 519-548, 2011.

KNIGHT, N. L.; WANG, J.; RIZOS, C. Generalised Measures Of Reliability For Multiple Outliers. Journal of Geodesy, v. 84, n. 10, p. 625-635, 2010.

MONICO, J. F. G.; MATSUOKA, M. T.; SAPUCCI, L. F. Confiabilidade interna e externa em Aplicações Geodésicas: Exemplo de uma Rede de Nivelamento. Geodésia Online, v. 2, p. 1, 2006.

MONICO, J. F. G. Posicionamento pelo GNSS: descrição, fundamentos $e$ aplicações. 2. ed. São Paulo: Ed. UNESP, 2008. 473p.

OBER, P.B. New, generally applicable metrics for RAIM/AAIM integrity monitoring. In: 9th international technical meeting of The Satellite Division of The Institute of Navigation, IONGPS-96, Kansas City, Missouri, p. 16771686, 1996.

OLIVEIRA, R.; DALMOLIN, Q. Critérios para a análise da geometria de redes geodésicas por componentes principais. Boletim de Ciências Geodésicas, v. 9, n. 1, p. 25-37, 2003.

OLIVEIRA, R.; DALMOLIN, Q. A Influência da Redundância da Observação sobre a Precisão dos Parâmetros. Boletim de Ciências Geodésicas, v. 14, n. 3, p. $295-315,2008$.

OLIVEIRA, R. Otimização dos pesos das observações geodésicas pelo problema de valor próprio inverso com considerações sobre o planejamento da confiabilidade da observação. 2007. Tese (Doutorado em Ciências Geodésicas) - Curso de Pós-Graduação em Ciências Geodésicas, Setor de Ciências da Terra, Universidade Federal do Paraná. Curitiba, 2007.

PEREZ, J. A. S.; MONICO, J. F. G.; CHAVES, J. C. Velocity Field Estimation Using GPS Precise Point Positioning: The South American Plate Case. Pesquisas. Instituto de Geociências da Universidade Federal do Rio Grande do Sul, v. 2, n. 2, p. 90-99, 2003.

PINTO, J. R. M. Potencialidade do uso do GPS em obras de Engenharia. Dissertação (mestrado). UNESP, Faculdade de Ciências e Tecnologia, Presidente Prudente, 2000.

TEIXEIRA, N. N.; FERREIRA, L. D. D. Análise da Confiabilidade de Redes Geodésicas. Boletim de Ciências Geodésicas, v. 9, n. 2, p. 199-216, 2003.

TEUNISSEN, P. J. G. Adjustment theory: an introduction. Delft: Ed. VSSD, 2003. $193 p$.

TEUNISSEN, P. J. G. Testing theory: an introduction. 2. ed. Delft: Ed. VSSD, 2006. 147 p.

(Recebido em junho de 2012. Aceito em agosto de 2012.) 